\title{
Single-Piece Solid-Contact Polymeric Membrane Ion-Selective Electrodes for Silver Ion
}

\author{
Tanji Yin,, ${ }^{\mathrm{a}, \mathrm{b}, \mathbf{c}, \mathbf{z}}$ Yuanyuan Liu, ${ }^{\mathrm{a}, \mathrm{d}}$ and Wei Qin ${ }^{\mathrm{a}}$ \\ ${ }^{a}$ Key Laboratory of Coastal Zone Environmental Processes and Ecological Remediation, Yantai Institute of Coastal \\ Zone Research (YIC), Chinese Academy of Sciences (CAS), Shandong Provincial Key Laboratory of Coastal Zone \\ Environmental Processes, YICCAS, Yantai, Shandong 264003, China \\ ${ }^{b}$ South China Sea Institute of Oceanology, Chinese Academy of Sciences, Guangzhou 510301, China \\ ${ }^{c}$ Graduate School of the Chinese Academy of Sciences, Beijing 100049, China \\ ${ }^{d}$ College of Chemistry and Biology, Yantai University, Yantai 264005, China
}

\begin{abstract}
Single-piece solid-contact polymeric membrane $\mathrm{Ag}^{+}$ion-selective electrodes (ISEs) have been fabricated based on conducting polymer poly(3-octylthiophene) (POT) dissolved into the $\mathrm{Ag}^{+}$ion-selective membrane. The effects of the amounts of POT on the potential responses of the single-piece solid-contact $\mathrm{Ag}^{+}$-ISEs were investigated in detail. Results indicate that the single-piece solid-contact $\mathrm{Ag}^{+}$-ISE with $10 \mathrm{wt} \%$ POT displays excellent reproducibility and stability of the potential response. The linear range of $3.0 \times 10^{-8}-3.0 \times 10^{-5} \mathrm{M}$ can be obtained in $\mathrm{AgNO}_{3}$ solution with the slope $56.84 \pm 0.92 \mathrm{mV} / \mathrm{dec}(n=3, R=0.9984)$ and the detection limit is $1.90 \times 10^{-8} \mathrm{M}$. In addition, the single-piece solid-contact $\mathrm{Ag}^{+}$-ISE with $10 \mathrm{wt} \% \mathrm{POT}$ shows excellent selectivity, stable potential response over a pH range of 3.5 to 6.0 and no significant redox sensitivity. The proposed electrode with $10 \mathrm{wt} \%$ POT has been successfully applied to potentiometric titration of chloride ion and determination of $\mathrm{Ag}^{+}$ion concentrations in spiked waters with high accuracy and good reliability.

(C) 2013 The Electrochemical Society. [DOI: 10.1149/2.004308jes] All rights reserved.
\end{abstract}

Manuscript submitted November 1, 2012; revised manuscript received February 20, 2013. Published April 25, 2013.

Silver is well known for its excellent antimicrobial property. It has been widely used in medicine, photography and production of ornaments. However, its potential damage to the environment has been easily neglected, although it is not as toxic to humans as other heavy metals. It has been reported that silver salts or silver nanoparticles show unacceptable toxic effects to the environment and human health. ${ }^{1}$ The U.S. Environmental Protection Agency reported that the amount of $\mathrm{Ag}^{+}$ion higher than $1.6 \mathrm{nM}$ in water is toxic to fish and microorganisms ${ }^{2}$ and the maximum contaminant level of total silver in drinking water is limited to $0.9 \mu \mathrm{M}^{3}$ Therefore, it is necessary to detect $\mathrm{Ag}^{+}$ion using efficient analytical methods.

Potentiometry with ion-selective electrodes (ISEs) is a promising method for directly determining various ions in clinical, environmental and industrial analysis, owning to their attractive features including simple design, small size, low energy consumption and low cost. Solid-contact ion-selective electrodes (ISEs) have recently attracted considerable attentions for simple fabrication, easy miniaturization and less maintenance, compared to the traditional polymeric membrane ISEs with inner filling solutions. ${ }^{4}$ Moreover, solid-contact ISEs can place in any position or configuration without the risk of leakage of the inner solution. However, the coated-wire electrodes (CWEs) ${ }^{5}$ as the initial solid-contact ISEs, suffer from the poor potential stability resulting from the blocked interface between the electronic conductor and the ion-selective membrane (ISM). ${ }^{6}$ Various materials have been used as solid-contact transducers to improve potential stability of solid-contact ISEs, including hydrogels, ${ }^{7}$ redox-active self-assembles monolayers, ${ }^{8}$ three-dimensionally ordered macroporous carbon, ${ }^{9}$ carbon nanotubes, ${ }^{10}$ fullerene, ${ }^{11}$ graphene, ${ }^{12}$ gold nanoparticles ${ }^{13}$ and conducting polymers. ${ }^{14-16}$ Among these materials, the conducting polymer poly(3-octylthiophene) (POT) is one of the most promising ion-to-transducers in solid-contact ISEs, because POT is less electroactive and may not take part in electrochemical side reactions to the same extent as the highly p-doped conducting polymers, such as poly(3,4-ethylenedioxythiophene). ${ }^{4}$ Moreover, POT is highly lipophilic, which may avoid the accumulation of water and salt between the electronic substrate and the ion-selective membrane. On the other side, single-piece solid-contact ISEs have been proposed by dispersing various electroactive materials into the ion-selective membranes. ${ }^{17-24}$ The fabrication procedures are much easier than that of the conventional solid-contact ISEs, which need the additional step to produce the intermediate layer. ${ }^{25,26}$
Here, we proposed a single-piece solid-contact polymeric membrane $\mathrm{Ag}^{+}$ion-selective electrode with conducting polymer poly(3octylthiophene) (POT) dissolved into the ion-selective membrane. The effects of POT concentrations on potential stability and selectivity were investigated in detail.

\section{Experimental}

Chemicals. - The ionophore $\quad o$-xylylene-bis $(N, \quad N$ diisobutyldithiocarbamate), sodium tetrakis[3,5-bis(trifluoromethyl) phenyl]borate (NaTFPB), high molecular weight poly(vinyl chloride) (PVC), 2-nitrophenyl octyl ether (o-NPOE) and regioregular poly(3octylthiophene-2,5-diyl) (POT) were purchased from Sigma-Aldrich.

All other chemicals were of analytical reagent grade. Deionized water (18.2 $\mathrm{M} \Omega \mathrm{cm}$ specific resistance) obtained with a Pall Cascada laboratory water system was used throughout.

Preparation of single-piece solid-contact $\mathrm{Ag}^{+}$ion-selective electrode.- The glassy carbon ( $\mathrm{GC}, \mathrm{CH}$ instruments, USA) electrodes with the diameter of $3 \mathrm{~mm}$ were used for fabrication of singlepiece solid-contact $\mathrm{Ag}^{+}$ion-selective electrodes. They were polished with $0.05 \mu \mathrm{m}$ alumina slurries, rinsed with deionized water and then ultrasonically cleaned.

The membrane components for single-piece solid-contact $\mathrm{Ag}^{+}$ ion-selective electrodes contained $0.77 \mathrm{wt} \%$ ionophore $(15 \mathrm{mmol} / \mathrm{kg})$, $0.44 \mathrm{wt} \%$ NaTFPB $(5 \mathrm{mmol} / \mathrm{kg}), 32.93 \mathrm{wt} \% \mathrm{PVC}$ and $65.86 \mathrm{wt} \%$ $o$-NPOE. POT $(3.6 \mathrm{mg} / \mathrm{mL})$ was prepared with chloroform. The membrane cocktail was prepared by dissolving $180 \mathrm{mg}$ of membrane components in $2 \mathrm{~mL}$ of tetrahydrofuran (THF) and stirred for $2 \mathrm{~h}$. Then a certain amount of POT solution was added into the membrane cocktail, which was kept under stirring for $1 \mathrm{~h}$ to form homogeneous mixture. The mixture were poured into a glass ring (i.d. $20 \mathrm{~mm}$ ) fixed on a glass plate. After overnight solvent evaporation, a disk of $5 \mathrm{~mm}$ diameter was punched from the membrane and then glued to the polished bare GC electrode with THF. Based on the different ratio between the weight of POT and that of the ionophore, the above prepared single-piece solid-contact electrodes are denoted as GC/Ag ${ }^{+}-\mathrm{ISM}(0 \% \mathrm{POT}), \mathrm{GC} \mathrm{Ag}^{+}-\mathrm{ISM}(1 \% \mathrm{POT}), \mathrm{GC} / \mathrm{Ag}^{+}-\mathrm{ISM}$ $(10 \%$ POT $), \mathrm{GC} \mathrm{Ag}^{+}-\mathrm{ISM}\left(50 \%\right.$ POT) and $\mathrm{GC} / \mathrm{Ag}^{+}-\mathrm{ISM}$ (100\% POT) electrodes, respectively. For the traditional potential measurements, the electrodes were conditioned for 1 day in $1.0 \times 10^{-4} \mathrm{M} \mathrm{AgNO}_{3}$. For the lower detection limit measurements, the electrodes were conditioned in $1.0 \times 10^{-4} \mathrm{M} \mathrm{AgNO}_{3}$ overnight and then in $1.0 \times 10^{-9} \mathrm{M} \mathrm{AgNO}_{3}$ for 2 days. For the selectivity 
measurements using the separate solution method (SSM), ${ }^{27}$ the electrodes were conditioned in $1.0 \times 10^{-3} \mathrm{M} \mathrm{NaNO}_{3}$ for 1 day.

Potentiometric measurements.- The potentiometric measurements were carried out using a CHI660C electrochemical workstation (Shanghai Chenhua Apparatus Co.) at room temperature in stirred solutions. The external reference electrode consisted of a doublejunction $\mathrm{Hg} / \mathrm{Hg}_{2} \mathrm{Cl}_{2}$ electrode with a $0.1 \mathrm{M} \mathrm{LiOAc}$ bridge electrolyte and saturated $\mathrm{KCl}$ solution as reference electrolyte. All potential values were corrected for liquid-junction potentials according to the Henderson equation and the ion activities were calculated by the DebyeHückel approximation.

Redox sensitivity of the single-piece solid-contact $\mathrm{Ag}^{+}$ISEs was carried out by measuring the potential response of the electrodes in $1 \mathrm{mM} \mathrm{Fe}(\mathrm{CN})_{6}^{3-/ 4-}$ redox couple with the ratio of $\mathrm{Fe}(\mathrm{III}) / \mathrm{Fe}$ (II) ranging from $1 / 10$ to $10 / 1$ at a constant ionic background of $0.1 \mathrm{M}$ $\mathrm{KCl}$ solution.

\section{Results and Discussion}

Selectivity of single-piece solid-contact $\mathrm{Ag}^{+}$ion-selective electrodes. - The selectivity of single-piece solid-contact ISEs are usually influenced by conducting polymers that are dissolved into the ion-selective membrane ${ }^{4}$. Therefore, separate solution method (SSM) is used to estimate the selectivity coefficients of the singlepiece solid-contact $\mathrm{Ag}^{+}$-ISEs in order to investigate the effect of POT. ${ }^{27}$ Fig. 1 shows the results of the selectivity coefficients of singlepiece solid-contact $\mathrm{Ag}^{+}-$ISEs with different amounts of POT. It can be seen that the selectivity coefficients of the GC/Ag ${ }^{+}-\mathrm{ISM}(1 \%$ POT) and $\mathrm{GC}_{\mathrm{Ag}}{ }^{+}-\mathrm{ISM}(10 \%$ POT) electrodes are slightly better than that of the GC/Ag ${ }^{+}-$ISM (0\% POT), except for $\mathrm{H}^{+}$and $\mathrm{Cu}^{2+}$ ions. The results may be due to the interaction between $\mathrm{Ag}^{+}$and sulfur atoms and $\pi$-coordination present in POT backbone, ${ }^{28}$ besides the interaction with the ionophore. However, the selectivity coefficients of the $\mathrm{GC} / \mathrm{Ag}^{+}-\mathrm{ISM}\left(50 \%\right.$ POT) and $\mathrm{GC} / \mathrm{Ag}^{+}-\mathrm{ISM}(100 \%$ POT) electrodes become poorer than that of the GC/Ag ${ }^{+}-\mathrm{ISM}(1 \%$ POT) and GC/Ag ${ }^{+}-$ISM (10\% POT) electrodes, which may be due to the fact that the excess of conducting polymer backbone influences the interaction between $\mathrm{Ag}^{+}$ion and the ionophore. Therefore, the $\mathrm{GC} / \mathrm{Ag}^{+}-\mathrm{ISM}(0 \% \mathrm{POT}), \mathrm{GC} / \mathrm{Ag}^{+}-\mathrm{ISM}(1 \% \mathrm{POT})$ and $\mathrm{GC} \mathrm{Ag}^{+}-\mathrm{ISM}(10 \%$ POT) electrodes were employed for the subsequent experiments. In addition, it's found that the selectivity of the proposed single-piece solid-contact $\mathrm{Ag}^{+}$-ISEs are much better than that of the reported $\mathrm{Ag}^{+}$-ISEs based on the chemically synthesized POT and [2.2.2] $p, p, p$-cyclophane as silver ionophore, ${ }^{28}$ which may be due to the fact that the proposed $\mathrm{Ag}^{+}$-ionophore with sulfur as coordinating sites shows much stronger interaction than that of cyclophane with $\pi$-coordination. ${ }^{29}$

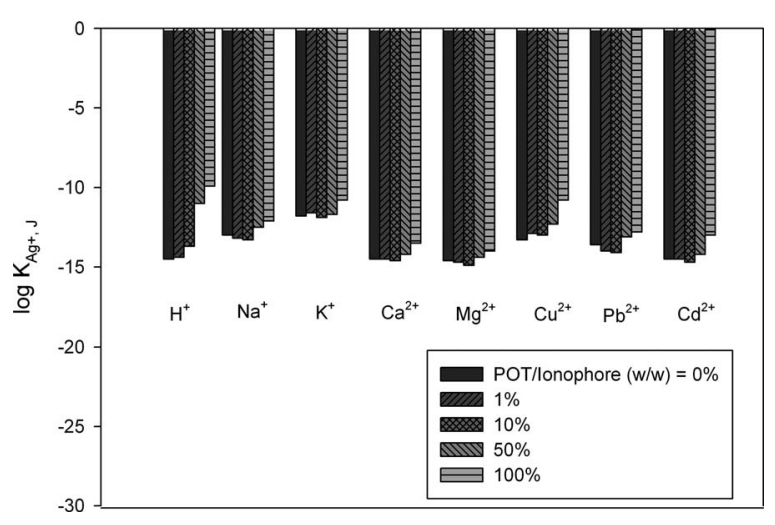

Figure 1. Potentiometric selectivity coefficients $\left(\log K_{A g}{ }^{+}, J^{P O T}\right)$ of singlepiece solid-contact $\mathrm{Ag}^{+}$ion-selective electrodes with the different ratio between the weight of POT and that of the ionophore using separate solution method (SSM).

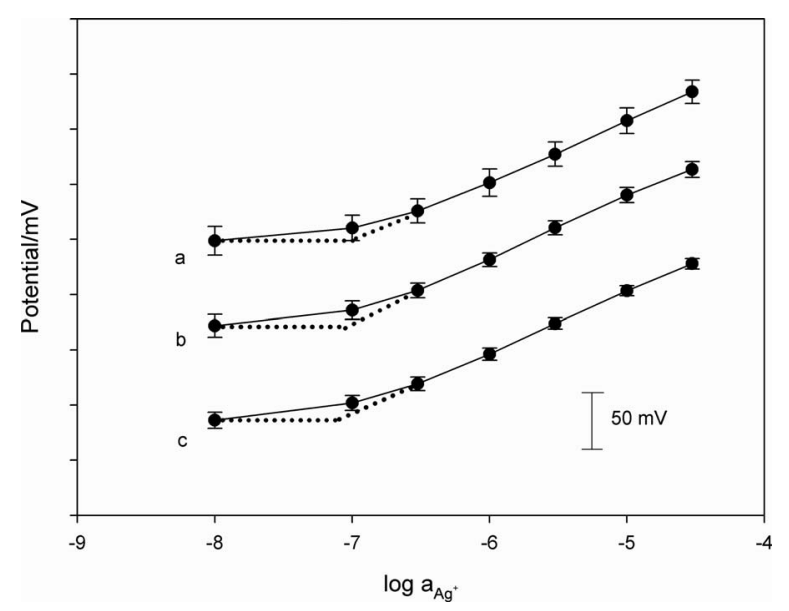

Figure 2. Calibration curves for (a) $\mathrm{GC} / \mathrm{Ag}^{+}-\mathrm{ISM}$ ( $0 \%$ POT), (b) $\mathrm{GC} / \mathrm{Ag}^{+}-\mathrm{ISM}(1 \% \mathrm{POT})$ and (c) $\mathrm{GC} / \mathrm{Ag}^{+}-\mathrm{ISM}$ (10\% POT) electrodes conditioned in $1.0 \times 10^{-4} \mathrm{M} \mathrm{AgNO}_{3}$ solution. Error bars represent the standard deviation for three identical electrodes.

Potentiometric response of single-piece solid-contact $\mathrm{Ag}^{+}$ionselective electrodes. - After conditioned in $1.0 \times 10^{-4} \mathrm{M} \mathrm{AgNO}_{3}$ solution overnight, the $\mathrm{GC} / \mathrm{Ag}^{+}-\mathrm{ISM}(0 \% \mathrm{POT}), \mathrm{GC} / \mathrm{Ag}^{+}-\mathrm{ISM}$ (1\% POT) and $\mathrm{GC} / \mathrm{Ag}^{+}-\mathrm{ISM}(10 \%$ POT) electrodes were measured in $\mathrm{AgNO}_{3}$ solution, respectively. As can be seen in Fig. 2, all of the single-piece solid-contact $\mathrm{Ag}^{+}$-ISEs with different amounts of POT exhibit the linear potential responses in the activity range of $3.0 \times 10^{-7}$ to $3.0 \times 10^{-5} \mathrm{M} \mathrm{AgNO}_{3}$. The slopes of the calibration curves are $54.53 \pm 1.75,54.97 \pm 0.74$ and $55.72 \pm 0.41 \mathrm{mV} / \mathrm{dec}$ and the detection limits calculated as the intersection of the two slope lines are $10^{-7.00}, 10^{-7.08}$ and $10^{-7.11} \mathrm{M}$ for the GC/Ag ${ }^{+}-\mathrm{ISM}$ (0\% POT), $\mathrm{GC} / \mathrm{Ag}^{+}-\mathrm{ISM}\left(1 \%\right.$ POT) and $\mathrm{GC} / \mathrm{Ag}^{+}-\mathrm{ISM}(10 \%$ POT) electrodes, respectively. In addition, it's found that the reproducibility of the $\mathrm{GC} / \mathrm{Ag}^{+}-\mathrm{ISM}(10 \%$ POT) electrode is much better than that of the GC/Ag ${ }^{+}-\mathrm{ISM}\left(0 \%\right.$ POT) and $\mathrm{GC} / \mathrm{Ag}^{+}-\mathrm{ISM}(1 \% \mathrm{POT})$ electrodes (see the error bars in Fig. 2). Therefore, the GC/Ag ${ }^{+}-\mathrm{ISM}$ (10\% POT) electrode would be used to detect $\mathrm{Ag}^{+}$ion with a lower detection limit.

In order to obtain the trace level measurements of $\mathrm{Ag}^{+}$ion, the

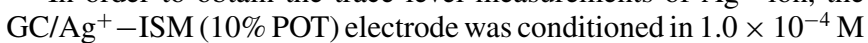
$\mathrm{AgNO}_{3}$ overnight and then in $1.0 \times 10^{-9} \mathrm{M} \mathrm{AgNO}_{3}$ for 2 days. Fig. 3 shows the calibration curve obtained by successively increasing $\mathrm{Ag}^{+}$

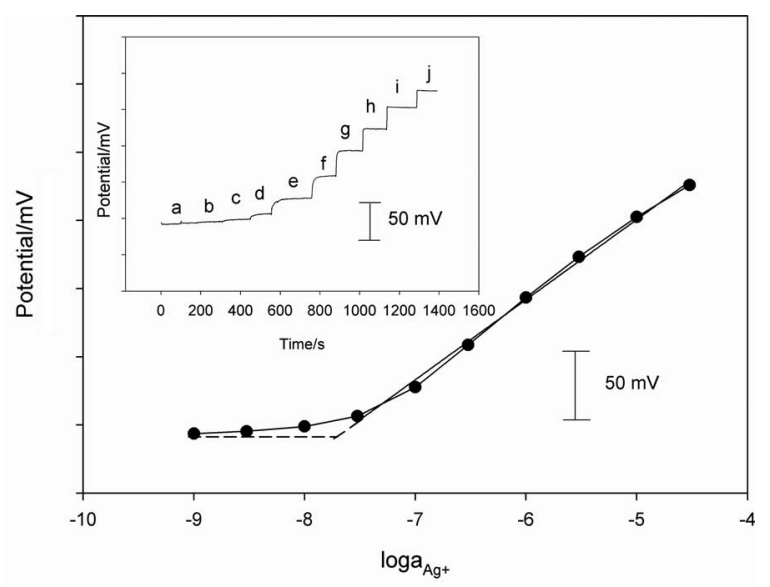

Figure 3. Calibration curve obtained with $\mathrm{GC} / \mathrm{Ag}^{+}-\mathrm{ISM}(10 \% \mathrm{POT})$ electrode conditioned in $1.0 \times 10^{-4} \mathrm{M} \mathrm{AgNO}_{3}$ overnight and then in $1.0 \times 10^{-9}$ $\mathrm{M} \mathrm{AgNO}_{3}$ for 2 days. Inset: Time traces of potential response of the singlepiece solid-contact $\mathrm{Ag}^{+}-\mathrm{ISE}$ (10\% POT). Concentrations of $\mathrm{Ag}^{+}$ion $(\mathrm{a}-\mathrm{j})$ : $1.0 \times 10^{-9}, 3.0 \times 10^{-9}, 1.0 \times 10^{-8}, 3.0 \times 10^{-8}, 1.0 \times 10^{-7}, 3.0 \times 10^{-7}, 1.0$ $\times 10^{-6}, 3.0 \times 10^{-6}, 1.0 \times 10^{-5}$ and $3.0 \times 10^{-5} \mathrm{M}$. 


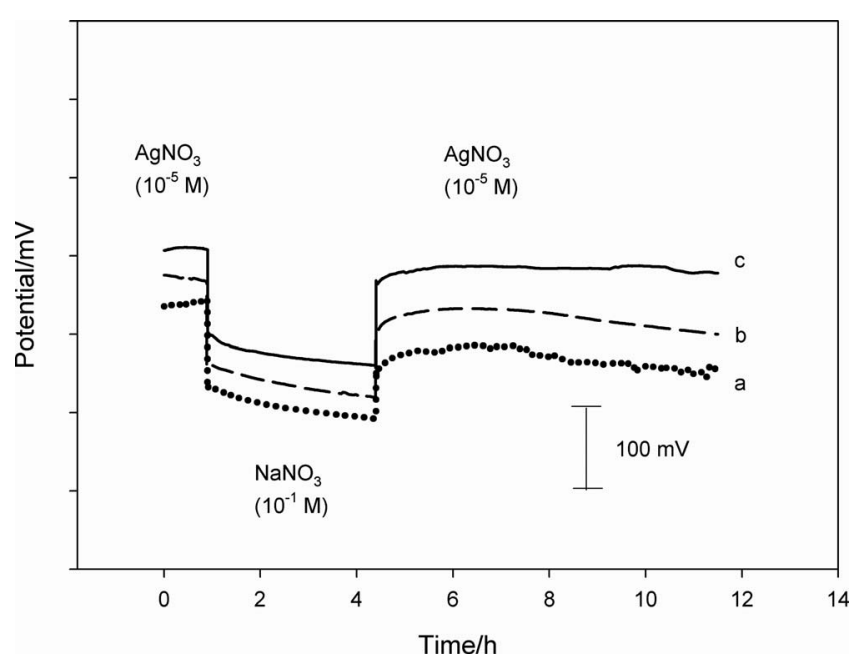

Figure 4. Water layer test for $\mathrm{GC} / \mathrm{Ag}^{+}-\mathrm{ISM}(0 \% \mathrm{POT})(\mathrm{a}), \mathrm{GC} / \mathrm{Ag}^{+}-\mathrm{ISM}$ (1\% POT) (b) and GC/Ag ${ }^{+}-\mathrm{ISM}(10 \%$ POT) (c) electrodes.

activities using the $\mathrm{GC} / \mathrm{Ag}^{+}-\mathrm{ISM}(10 \%$ POT) electrode. The linear range of $3.0 \times 10^{-8}-3.0 \times 10^{-5} \mathrm{M}$ was obtained with the slope 56.84 $\pm 0.92 \mathrm{mV} / \mathrm{dec}(n=3, R=0.9984)$. The detection limit is $(1.90$ $\pm 0.08) \times 10^{-8} \mathrm{M}$, which is much lower than that of the POT-based potentiometric $\mathrm{Ag}^{+}$ion sensors. ${ }^{28}$ In addition, the response time is less than $20 \mathrm{~s}$ (see inset of Fig. 3).

Water layer test.- For long-term measurements of solid-contact ISEs, the formation of a thin aqueous layer at the interface between the electronic substrate and ion-selective membrane would lead to the instability of potential. Therefore, the effect of water layer on potential was investigated by the potentiometric water layer test. ${ }^{6}$ After conditioned in $1.0 \times 10^{-4} \mathrm{M} \mathrm{AgNO}_{3}$ solution overnight, the $\mathrm{GC} / \mathrm{Ag}^{+}-\mathrm{ISM}$ (0\% POT), GC/Ag ${ }^{+}-\mathrm{ISM}$ (1\% POT) and GC/Ag ${ }^{+}-\mathrm{ISM}(10 \%$ POT) electrodes were alternately measured in $1.0 \times 10^{-5} \mathrm{M} \mathrm{AgNO}_{3}$ for $1 \mathrm{~h}, 0.1 \mathrm{M} \mathrm{NaNO}_{3}$ for $3.5 \mathrm{~h}$ and again $1.0 \times 10^{-5} \mathrm{M} \mathrm{AgNO}_{3}$ for 7 h. As seen in Fig. 4, the GC/Ag ${ }^{+}-$ISM (10\% POT) electrode shows potential drift less than $1.1 \pm 0.05 \mathrm{mV} / \mathrm{h}$ after the sample solution is changed from $0.1 \mathrm{M} \mathrm{NaNO}_{3}$ to $1.0 \times 10^{-5} \mathrm{M} \mathrm{AgNO}_{3}$ solution, while the potential changes are equal to $4.88 \pm 0.20$ and 4.57 $\pm 0.14 \mathrm{mV} / \mathrm{h}$ for the $\mathrm{GC} / \mathrm{Ag}^{+}-\mathrm{ISM}\left(0 \%\right.$ POT) and $\mathrm{GC} / \mathrm{Ag}^{+}-\mathrm{ISM}$

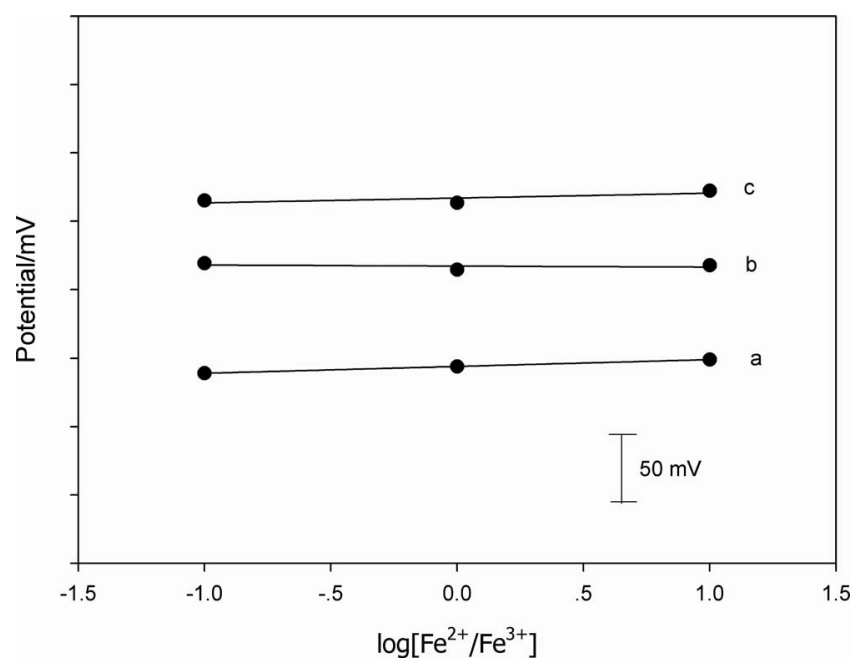

Figure 5. Redox sensitivity of GC/Ag ${ }^{+}-\mathrm{ISM}\left(0 \%\right.$ POT) (a), GC/Ag ${ }^{+}-\mathrm{ISM}$ (1\% POT) (b) and $\mathrm{GC} / \mathrm{Ag}^{+}-\mathrm{ISM}(10 \%$ POT) (c) electrodes.

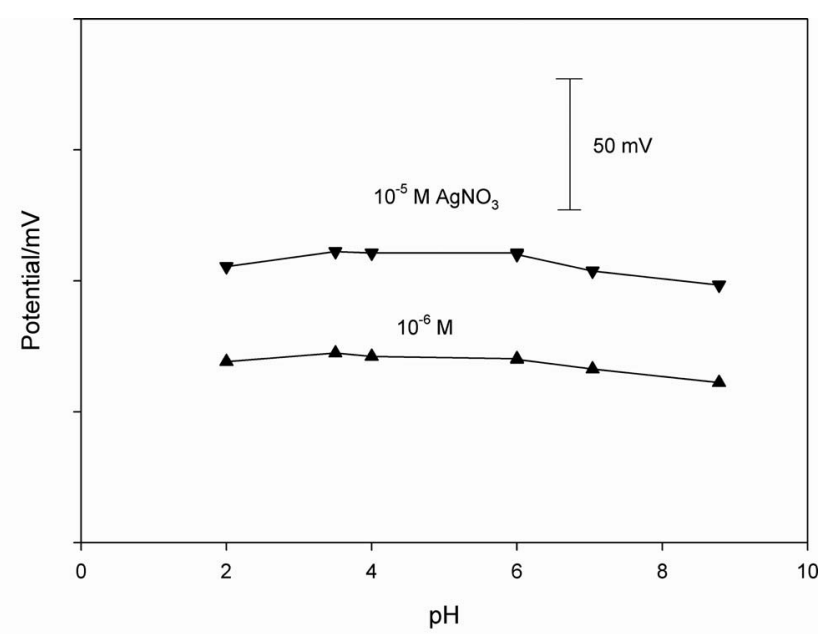

Figure 6. Effect of $\mathrm{pH}$ on the potential responses of the GC/Ag ${ }^{+}-\mathrm{ISM}(10 \%$ POT) electrode in $1.0 \times 10^{-6}$ and $1.0 \times 10^{-5} \mathrm{M} \mathrm{AgNO}_{3}$ with $0.1 \mathrm{M} \mathrm{NaNO}_{3}$ as a constant ionic background.

(1\% POT) electrodes, respectively. Therefore, the $\mathrm{GC} / \mathrm{Ag}^{+}-\mathrm{ISM}$ (10\% POT) electrode shows more stable potential response.

Redox sensitivity. - The redox sensitivity measurements were investigated for the $\mathrm{GC} / \mathrm{Ag}^{+}-\mathrm{ISM}\left(0 \%\right.$ POT), GC/Ag ${ }^{+}-\mathrm{ISM}(1 \%$ POT) and $\mathrm{GC} / \mathrm{Ag}^{+}-\mathrm{ISM}$ (10\% POT) electrodes, respectively. As shown in Fig. 5, all of the single-piece solid-contact $\mathrm{Ag}^{+}-$ISEs with different amounts of POT show no significant redox sensitivity, which may be due to the fact that PVC-based ion-selective membrane is an electronic insulator and undoped POT is an electronic semiconductor with relatively low conductivity. ${ }^{30}$

pH effect.- The effect of $\mathrm{pH}$ on the potential response of the

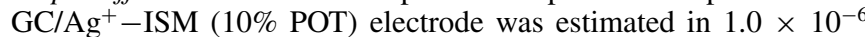
and $1.0 \times 10^{-5} \mathrm{M} \mathrm{AgNO}_{3}$ at a constant ionic background of $0.1 \mathrm{M}$ $\mathrm{NaNO}_{3}$ solution. The $\mathrm{pH}$ of the solutions was adjusted using $\mathrm{HNO}_{3}$ or $\mathrm{NaOH}$. As shown in Fig. 6, the potential of the GC/Ag ${ }^{+}-\mathrm{ISM}(10 \%$ POT) electrode remains almost constant over a $\mathrm{pH}$ range of 3.5 to 6.0. The potential change at lower $(<3.5)$ and higher $(>6.0) \mathrm{pH}$ values could be due to the protonation of the ionophore and the formation of silver hydroxide, ${ }^{31}$ respectively.

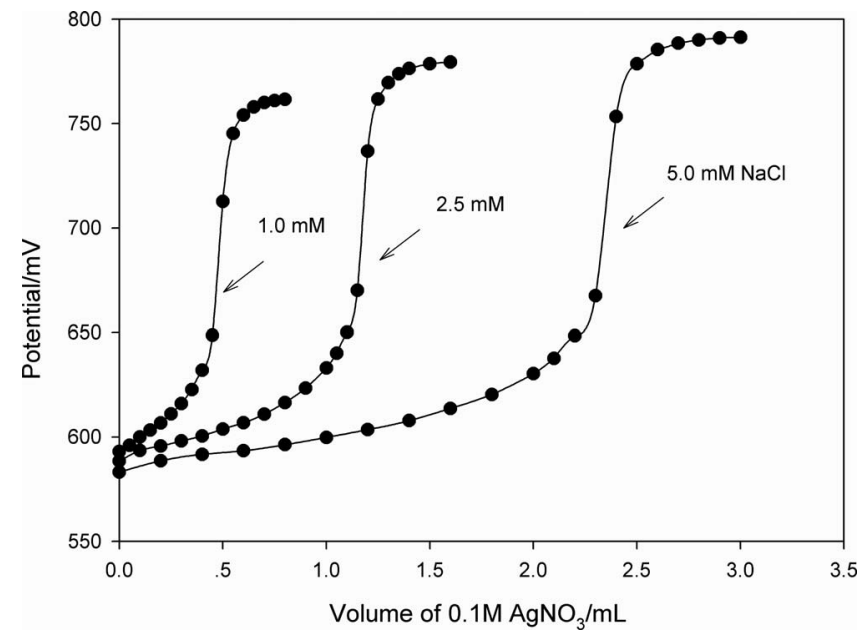

Figure 7. Titration curves of $50.0 \mathrm{~mL}$ of $1.0,2.5,5.0 \mathrm{mM} \mathrm{NaCl}$ solutions with $0.1 \mathrm{M} \mathrm{AgNO}_{3}$ as titration reagent obtained by using $\mathrm{GC}_{\mathrm{Ag}}{ }^{+}-\mathrm{ISM}(10 \% \mathrm{POT})$ electrode as indicator electrode. 


\begin{tabular}{cccc}
\hline $\begin{array}{l}\text { Table I. Application of the proposed GC/Ag } \\
\text { detect } \mathrm{Ag}^{+} \text {ion in spiked waters. }\end{array}$ & & \\
Sample & Added $(\mu \mathrm{M})$ & Found & \\
& $\mathrm{a}(\mu \mathrm{M})$ & Recovery $(\%)$ \\
\hline Tap water 1 & 0 & $\mathrm{ND}^{\mathrm{b}}$ & - \\
& 0.100 & $0.103 \pm 0.008$ & 103.0 \\
& 0.300 & $0.285 \pm 0.015$ & 95.0 \\
Tap water 2 & 0 & $\mathrm{ND}^{\mathrm{b}}$ & - \\
& 0.050 & $0.048 \pm 0.005$ & 96.0 \\
Lake water 1 & 0.100 & $0.094 \pm 0.006$ & 94.0 \\
& 0 & $\mathrm{ND}^{\mathrm{b}}$ & - \\
& 0.100 & $0.109 \pm 0.010$ & 109.0 \\
& 0.300 & $0.299 \pm 0.008$ & 99.7
\end{tabular}

${ }^{\mathrm{a}}$ Mean of three determinations \pm S.D.

${ }^{\mathrm{b}}$ Not detected.

Analytical applications. - The GC/Ag ${ }^{+}$- ISM (10\% POT) electrode was successfully applied as an indicator electrode in the potentiometric titration of $\mathrm{NaCl}$ solution with $\mathrm{AgNO}_{3}$. Fig. 7 shows the typical titration curves of $1.0,2.5,5.0 \mathrm{mM} \mathrm{NaCl}$ solutions with $0.1 \mathrm{M} \mathrm{AgNO}_{3}$ as titration reagent. It can be seen that obvious inflection points are observed in the titration curves, which indicates that the amount of $\mathrm{Cl}^{-}$ion in the solution can be accurately determined with the perfect stoichiometry by using GC/Ag ${ }^{+}-\mathrm{ISM}(10 \%$ POT) electrode.

The application of the GC/Ag ${ }^{+}$- ISM (10\% POT) electrode was further tested by measuring $\mathrm{Ag}^{+}$ion concentrations in spiked waters using standard addition potentiometric method. Table I shows that recoveries vary from 94.0 to $109.0 \%$, which indicate that the proposed GC/Ag ${ }^{+}-\mathrm{ISM}$ (10\% POT) electrode can be successfully applied to detect $\mathrm{Ag}^{+}$ion in real samples with high accuracy and good reliability.

\section{Conclusions}

Single-piece solid-contact polymeric membrane $\mathrm{Ag}^{+}$ion-selective electrodes have been successfully developed using conducting polymer poly(3-octylthiophene) (POT) dissolved into the ion-selective membrane. The potential responses of the electrodes are significantly influenced by the amounts of POT in the ion-selective membrane. Results indicate that the single-piece solid-contact $\mathrm{Ag}^{+}$ion-selective electrode with $10 \mathrm{wt} \%$ POT displays excellent potential reproducibility, stability and selectivity. The calibration curve can be obtained in $\mathrm{AgNO}_{3}$ solution using the proposed electrode with $10 \mathrm{wt} \%$ POT in the linear range of $3.0 \times 10^{-8}-3.0 \times 10^{-5} \mathrm{M}$ with the slope $56.84 \pm 0.92 \mathrm{mV} / \mathrm{dec}(n=3, R=0.9984)$ and the detection limit is $1.9 \times 10^{-8} \mathrm{M}$. Moreover, the electrode exhibits no significant redox sensitivity, which is attributed to the electronic insulated PVC-based ion-selective membrane and undoped POT with low conductivity. The proposed electrode with $10 \mathrm{wt} \%$ POT has been successfully applied to potentiometric titration of chloride ion and determination of $\mathrm{Ag}^{+}$ion concentrations in spiked waters with high accuracy and good reliabil- ity. The proposed sensor provides a promising method for fabrication of a simple, robust and reliable $\mathrm{Ag}^{+}$-ISE that can be miniaturized and applied in many fields to detect $\mathrm{Ag}^{+}$ion.

\section{Acknowledgments}

This work was financially supported by the Outstanding Youth National Science Foundation of Shandong Province (JQ200814), the National Natural Science Foundation of China (20977073) and the Taishan Scholar Program of Shandong Province (TS20081159).

\section{References}

1. N. R. Panyala, E. M. Pena-Mendez, and J. Havel, J. Appl. Biomed., 6, 117 (2008), http://www.zsf.jcu.cz/jab/6_3/havel6_3.htm.

2. E. p. agency, in EPA drinking water criteria document for silver, EPS CASRN 7440 7422-7444, Washington, DC (1989)

3. E. p. agency, in National primary drinking water regulations: final rule, Fed. Regist. 56: 3526, Washington, DC (1991).

4. J. Bobacka, A. Ivaska, and A. Lewenstam, Chem. Rev., 108, 329 (2008).

5. R. W. Cattrall and H. Freiser, Anal. Chem., 43, 1905 (1971).

6. M. Fibbioli, W. E. Morf, M. Badertscher, N. F. de Rooij, and E. Pretsch, Electroanalysis, 12, $1286(2000)$

7. A. Lynch, D. Diamond, and M. Leader, Analyst, 125, 2264 (2000).

8. E. Grygolowicz-Pawlak, K. Plachecka, Z. Brzozka, and E. Malinowska, Sens. Actuators, $B, \mathbf{1 2 3}, 480$ (2007)

9. M. A. Fierke, C.-Z. Lai, P. Bühlmann, and A. Stein, Anal. Chem., 82, 680 (2009).

10. G. A. Crespo, S. Macho, and F. X. Rius, Anal. Chem., 80, 1316 (2008).

11. M. Fouskaki and N. Chaniotakis, Analyst, 133, 1072 (2008).

12. J. Ping, Y. Wang, J. Wu, and Y. Ying, Electrochem. Commun., 13, 1529 (2011).

13. E. Jaworska, M. Wojcik, A. Kisiel, J. Mieczkowski, and A. Michalska, Talanta, 85, 1986 (2011).

14. S. Y. Yu, F. H. Li, T. J. Yin, Y. M. Liu, D. W. Pan, and W. Qin, Anal. Chim. Acta, 702, 195 (2011)

15. J. Larrieux, O. Abrou, and K. Levon, Macromol. Symp., 304, 8 (2011).

16. S. Anastasova-Ivanova, U. Mattinen, A. Radu, J. Bobacka, A. Lewenstam, J. Migdalski, M. Danielewski, and D. Diamond, Sens. Actuators, B, 146, 199 (2010).

17. J. Bobacka, T. Lindfors, M. McCarrick, A. Ivaska, and A. Lewenstam, Anal. Chem., 67, 3819 (1995).

18. T. Lindfors, S. Ervelä, and A. Ivaska, J. Electroanal. Chem., 560, 69 (2003).

19. A. L. Grekovich, N. N. Markuzina, K. N. Mikhelson, M. Bochenska, and A. Lewenstam, Electroanalysis, 14, 551 (2002).

20. R. Toczyłowska, R. Pokrop, A. Dybko, and W. Wróblewski, Anal. Chim. Acta, 540, 167 (2005).

21. J. Zhu, Y. Qin, and Y. Zhang, Electrochem. Commun., 11, 1684 (2009).

22. J. Zhu, X. Li, Y. Qin, and Y. Zhang, Sens. Actuators, B, 148, 166 (2010).

23. P. Sjöberg-Eerola, J. Bobacka, T. Sokalski, J. Mieczkowski, A. Ivaska, and A. Lewenstam, Electroanalysis, 16, 379 (2004).

24. S. Xu, Q. Yuan, S. Yu, and X. Wu, Electroanalysis, 24, 2021 (2012).

25. K. Y. Chumbimuni-Torres, N. Rubinova, A. Radu, L. T. Kubota, and E. Bakker, Anal. Chem., 78, 1318 (2006).

26. C.-Z. Lai, M. Joyer, M. Fierke, N. Petkovich, A. Stein, and P. Bühlmann, J. Solid State Electrochem., 13, 123 (2009).

27. E. Bakker, J. Electrochem. Soc., 143, L83 (1996).

28. M. Vázquez, J. Bobacka, and A. Ivaska, J. Solid State Electrochem., 9, 865 (2005).

29. Z. Szigeti, A. Malon, T. Vigassy, V. Csokai, A. Grün, K. Wygladacz, N. Ye, C. Xu, V. J. Chebny, I. Bitter, R. Rathore, E. Bakker, and E. Pretsch, Anal. Chim. Acta, 572, 1 (2006).

30. P. Sjöberg-Eerola, J. Bobacka, A. Lewenstam, and A. Ivaska, Sens. Actuators, B, 127, 545 (2007).

31. A. Demirel, A. Doğan, G. Akkuş, M. Yılmaz, and E. Kılıç, Electroanalysis, 18, 1019 (2006). 